\title{
A prospective phase I dose-escalation trial of stereotactic ablative radiotherapy (SABR) as an alternative to cytoreductive nephrectomy for inoperable patients with metastatic renal cell carcinoma
}

\author{
Rohann J. M. Correa', Belal Ahmad' ${ }^{1}$, Andrew Warner' ${ }^{1}$ Craig Johnson'1, Mary J. MacKenzie², Stephen E. Pautler ${ }^{3,4}$, \\ Glenn S. Bauman', George B. Rodrigues ${ }^{1,5}$ and Alexander V. Louie ${ }^{1,5^{*}}$
}

\begin{abstract}
Background: Cytoreductive nephrectomy is thought to improve survival in metastatic renal cell carcinoma (mRCC). As many patients are ineligible for major surgery, we hypothesized that SABR could be a safe alternative.

Methods: In this dose-escalation trial, inoperable mRCC patients underwent SABR targeting the entire affected kidney. Toxicity (CTCAE v3.0), quality of life (QoL), renal function, and tumour response (RECIST v1.0) were assessed.

Results: Twelve patients of mostly intermediate (67\%) or poor (25\%) International Metastatic Renal Cell Carcinoma Database Consortium (IMDC) prognostic class, median KPS of 70\%, and median tumour size of $8.7 \mathrm{~cm}$ (range: 4.8-13.8) were enrolled in successive dose cohorts of $25(n=3), 30(n=6)$, and $35 \mathrm{~Gy}(n=3)$ in 5 fractions. SABR was well tolerated with 3 grade 3 events: fatigue (2) and bone pain (1). QoL decreased for physical well-being $(p=0.016)$, but remained unchanged in other domains. SABR achieved a median tumour size reduction of $-17.3 \%$ (range: +5.3 to -54.4 ) at 5.3 months. All patients progressed systemically and median OS was 6.7 months. Crude median follow-up was 5.8 months.
\end{abstract}

Conclusions: In non-operable mRCC patients, renal-ablative SABR to 35 Gy in 5 fractions yielded acceptable toxicity, renal function preservation, and stable QoL. SABR merits further prospective investigation as an alternative to cytoreductive nephrectomy.

Trial Registration: ClinicalTrials.gov NCT02264548. Registered July 222014 - Retrospectively registered: https://clinicaltrials. gov/ct2/show/NCT02264548

Keywords: Primary tumour, Renal cell, Metastatic, Stereotactic ablative radiotherapy

\section{Background}

Metastatic renal cell carcinoma (mRCC) represents one of the few clinical scenarios in which randomized evidence supports aggressive primary tumour control via cytoreductive nephrectomy $(\mathrm{CN})$, as this is associated with improved overall survival when followed by interferon-based systemic

\footnotetext{
* Correspondence: dr.alexlouie@gmail.com

'Department of Radiation Oncology, London Regional Cancer Program, London, Canada

${ }^{5}$ Department of Epidemiology and Biostatistics, Western University, London, Canada

Full list of author information is available at the end of the article
}

therapy $[1,2]$. Likewise, non-randomized evidence in the era of tyrosine kinase inhibitors (TKI) also supports a survival benefit of $\mathrm{CN}$ in select mRCC patients $[3,4]$ although recent evidence supports superior overall survival with upfront TKI and deferred $\mathrm{CN}$ vs. immediate $\mathrm{CN}$ [5]. However, rates of $\mathrm{CN}$ have declined in the TKI era [6,7]; moreover, poor-PS and poor-risk patients do not benefit from $\mathrm{CN}[4]$ as a result of greater post-surgical morbidity [8].

Stereotactic ablative radiotherapy (SABR) is emerging as an effective ablative modality for renal tumours. Although $\mathrm{RCC}$ is considered resistant to standard fractionation radiotherapy [9], hypofractionated radiotherapy yields histologic 
tumour ablation [10, 11], and clinically, SABR is highly effective in treating RCC oligometastases and can postpone or obviate the need for systemic therapy in some instances $[12,13]$. Likewise, SABR is also an emerging ablative modality for primary RCC tumours, achieving high rates of local control with minimal toxicity $[14,15]$. In a recent multi-institutional pooled analysis conducted by the International Radiosurgery Oncology Consortium for Kidney (IROCK), 223 patients were treated with SABR targeting RCC primary tumours, yielding a local control rate of $97.8 \%$ at 2 years with CTCAE $\geq$ Gr. 3 toxicity rate of $1.3 \%$ [16]. While alternative ablative therapies such as cryotherapy or radiofrequency ablation can target primary tumours, these are limited by size $(<4 \mathrm{~cm})$ and location (peripheral) $[17,18]$.

We hypothesized that SABR may represent an alternative ablative modality for those patients who may otherwise have benefited from cytoreductive nephrectomy, but are medically inoperable or have unresectable primary tumours. Moreover, given recent evidence that SABR may stimulate anti-tumour immunity to increase sensitivity to contemporary immunotherapy [19-21], there is strong rationale to establish the safety of this technique, permitting its combination with immune checkpoint inhibitors to potentially enhance their effectiveness.

We previously published our retrospective experience [22] wherein SABR safely and tolerably treated unconventionally large renal tumours (median $9.5 \mathrm{~cm}$, above the concensus 5-8 cm upper limit of size [23]). Here we report the results of a prospective trial of 5 -fraction SABR targeting the tumour-bearing kidney in mRCC. The primary endpoint was safety/toxicity and secondary endpoints included renal function, patient-reported quality of life, tumour response, and overall survival.

\section{Methods}

\section{Patient Eligibility}

In this ethics board-approved protocol (Western University REB\# 15680), eligible patients were $\geq 18$ years old, were diagnosed with stage IV, biopsy-confirmed RCC, and were deemed medically inoperable or harbored an unresectable primary tumour. Operability was assessed by a urologic oncologist (all patients were reviewed in a multi-disciplinary clinic including a surgeon, radiation oncologist, and medical oncologist). Patients could be enrolled regardless of systemic therapy status and could receive SABR before or after. Enrolled patients underwent detailed pre-screening including baseline performance status and QoL assessment, bloodwork, renal perfusion scan, and CT imaging. Those with bilateral renal involvement, poor baseline renal function (creatinine clearance $<50 \mathrm{~mL} / \mathrm{min}$ or $<40 \%$ flow to the contralateral kidney on renal perfusion scan), non-RCC histology, or life expectancy $<8$ weeks were excluded. Patients receiving systemic therapy were included if treatment was discontinued at least 2 weeks prior to SABR. Detailed inclusion and exclusion criteria are provided on ClinicalTrials.gov (NCT02264548).

\section{Dose Escalation Protocol}

The primary endpoint of this trial was safety/toxicity, evaluated via the dose escalation cohort schema depicted in Fig. 1. Each dose cohort was monitored during treatment and in follow-up for dose limiting toxicities (DLTs), defined as any CTCAE v 3.0 grade 3,4 , or 5 toxicity event that was definitely/probably/possibly related to treatment. Recruitment of at least 3 patients to each dose level $(25,30,35$, and 40 Gy in 5 daily fractions) was planned. Advancement to the subsequent dose level was permitted in the absence of a DLT up to 4 weeks postSABR. If a DLT event occurred in a cohort, that dose level was repeated with 3 additional patients. If no further DLTs occurred in the repeat cohort, recruitment to the next dose level was permitted. The maximum tolerated dose (MTD) was reached if a second DLT occurred.

Due to a single DLT that occurred in the 30 Gy cohort, a repeat cohort at that dose level was recruited. MTD for this trial was reached when two DLT events were reported in the 35 Gy cohort. Thus, no patients were recruited to the 40 Gy cohort.

\section{Treatment Technique}

All enrolled patients were immobilized in a supine position using a vacuum body mould as per institutional standards. Motion control was achieved using four-dimensional CT simulation wherein images were obtained at all phases of the respiratory cycle to generate a composite integrated target volume. OARs were contoured (without expansion for planning risk volume (PRV)) and dose limited to these structures based on established constraints [23, 24]. The

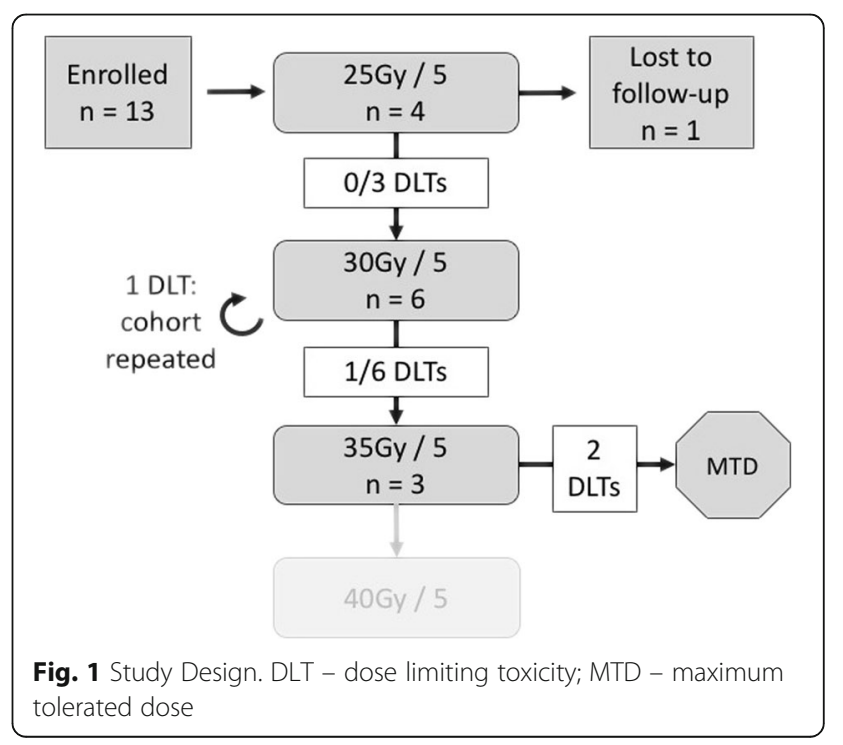


clinical target volume (CTV) included the primary tumour and ipsilateral kidney to recapitulate a cytoreductive nephrectomy. The initial planning target volume (PTV-I) was defined as the CTV with a $5 \mathrm{~mm}$ expansion in all three dimensions, whereas the final PTV (PTV-F) was a compromised PTV generated by subtracting any small bowel OAR volume from the PTV-I (Additional file 1: Figure S1). Dose was prescribed to the PTV-F.

\section{Follow-up and Evaluation of Clinical Outcomes}

Patients were monitored for adverse events on each treatment day, then at 4,8 , and 12 weeks, and subsequently at 6-month intervals thereafter. Follow-up visits included clinical assessment of toxicity using the Common Terminology Criteria for Adverse Events version 3.0 (CTCAE v4.0), QoL (FACT-G \& FACT-KSI) and general symptom assessment (ESAS) using standard questionnaires, bloodwork (CBC, electrolytes, urea, creatinine, calcium, albumin, liver enzymes, bilirubin, alkaline phosphatase) and urinalysis. Pre- and post-SABR creatinine clearance $(\mathrm{CrCl})$ was calculated using the Cockroft-Gault equation and glomerular filtration rate (GFR) measured using nuclear medicine renal perfusion scans. Post-SABR CT scans were performed at 4, 8, and 12 weeks and then at 6 month intervals post-SABR to assess tumour response.

\section{Statistical Analysis}

Descriptive statistics were generated for baseline patient and disease characteristics. The change in creatinine $\mathrm{CrCl}$, GFR, or QoL scores pre- and post-SABR were evaluated using the Wilcoxon signed rank test. A Kaplan-Meier estimate of overall survival including 95\% confidence bands was generated for all patients. All statistical analysis was performed using SAS version 9.4 software (SAS institute, Cary, NC), using 2-sided statistical testing at the 0.05 significance level.

\section{Results}

\section{Patient and Disease Characteristics}

From July 2009 to June 2016, 13 patients were enrolled in the trial beginning at the 25 Gy dose level and proceeding as depicted in Fig. 1. One patient from the 25 Gy cohort was lost to follow-up prior to the first post-treatment visit and thus was excluded. No acute toxicities were recorded for this patient while on treatment. As summarized in Table 1 , the remaining 12 patients were of median age 66.8 years (range: $55-85)$, median KPS of $70 \%$ (40-90\%), and were mostly of 'Intermediate' (66.7\%) or 'Poor' (25\%) International Metastatic Renal Cell Carcinoma Database Consortium (IMDC) prognostic class [25]. The majority of tumours were T2-T4 (91.7\%), biopsy-proven RCC (clear cell histology in $75 \%$ ), and $8.7 \mathrm{~cm}$ median size (4.8-13.8). One patient's tumour histology was "poorly differentiated carcinoma" but was deemed eligible given the clinical context.
Table 1 Patient Characteristics $(N=12)$

\begin{tabular}{|c|c|}
\hline Characteristic & Value \\
\hline Age at diagnosis - median (range) & $66.8(55.0-85.1)$ \\
\hline Male - n (\%) & $7(58.3)$ \\
\hline \multicolumn{2}{|l|}{ Laterality - n (\%) } \\
\hline Left Kidney & $4(33.3)$ \\
\hline Right Kidney & $8(66.7)$ \\
\hline \multicolumn{2}{|l|}{ Histology - n (\%) } \\
\hline Clear Cell & $9(75)$ \\
\hline Papillary & $2(16.7)$ \\
\hline Other $^{\mathrm{a}}$ & $1(8.3)$ \\
\hline \multicolumn{2}{|l|}{ Location of Metastases $^{b}$} \\
\hline Lung & $10(83.3)$ \\
\hline Bone & $6(50)$ \\
\hline Lymph nodes & $5(41.7)$ \\
\hline Brain & $3(25)$ \\
\hline Other $^{c}$ & $4(33.3)$ \\
\hline Karnofsky Performance Status - median (range) & $70(40-90)$ \\
\hline \multicolumn{2}{|l|}{ Karnofsky Performance Status - n (\%) } \\
\hline$\geq 80 \%$ & $5(41.7)$ \\
\hline$<80 \%$ & $7(58.3)$ \\
\hline \multicolumn{2}{|l|}{ IMDC Prognostic Group - n (\%) } \\
\hline Favorable & $1(8.3)$ \\
\hline Intermediate & $8(66.7)$ \\
\hline Poor & $3(25)$ \\
\hline \multicolumn{2}{|l|}{ Systemic Therapy Timing ${ }^{\mathrm{b}}-\mathrm{n}(\%)$} \\
\hline None & $6(50)$ \\
\hline Pre-SABR & $1(8.3)$ \\
\hline Post-SABR & $4(33.3)$ \\
\hline Both & $1(8.3)$ \\
\hline \multicolumn{2}{|l|}{ Systemic Therapy Type - n (\%) } \\
\hline None & $6(50)$ \\
\hline Pazopanib or Sunitinib & $5(41.7)$ \\
\hline Temsirolimus or Everolimus & $2(16.7)$ \\
\hline \multicolumn{2}{|l|}{ SABR Treatment Technique - $\mathrm{n}(\%)$} \\
\hline VMAT & $7(58.3)$ \\
\hline TOMO & $5(41.7)$ \\
\hline \multicolumn{2}{|l|}{ Dose Cohort (Gy) - n (\%) } \\
\hline $25 / 5$ & $3(25)$ \\
\hline $30 / 5$ & $6(50)$ \\
\hline $35 / 5$ & $3(25)$ \\
\hline Primary tumor size $(\mathrm{cm})-$ median (range) & $8.7(4.8-13.8)$ \\
\hline Actuarial median follow-up (months) ${ }^{e}$ - median $(95 \%$ Cl) & $22(4.63, \mathrm{~N} / \mathrm{A})$ \\
\hline
\end{tabular}

IMDC: International Metastatic Renal Cell Carcinoma Database Consortium, SABR: stereotactic ablative radiotherapy, Cl: confidence interval, TOMO: helical tomotherapy, IMRT: intensity modulated radiotherap. ${ }^{\mathrm{a}}$ Poorly-differentiated carcinoma $(n=1) ;{ }^{\text {b }}$ Categories not mutually-exclusive; ' Kidney $(n=1)$, liver $(n=2)$, left adrenal $(n=1)$; ${ }^{\mathrm{d}}$ Longest tumor dimension on CT scan; ${ }^{\mathrm{e}}$ Reverse Kaplan-Meier Method 
Median CTV and PTV-F were $535.0 \mathrm{~cm}^{3}$ (288.2-883.5) and $763.1 \mathrm{~cm}^{3}$ (264.9-1234.4), respectively. Distant metastases occurred most commonly in lung, bone, and brain. Six of the 12 patients received systemic therapy pre-SABR (1), post-SABR (4), or both (1). Systemic therapy was initiated any time after SABR (given a minimum 2 week washout period) as indicated for progression of metastatic disease. Additional file 2: Figure S2 depicts the timing of systemic therapy, per patient, relative to completion of SABR. Six patients did not receive systemic therapy.

\section{Treatment Parameters}

Doses to treatment volumes and OAR are listed by individual patient in Additional file 3: Table S1. Favorable coverage of treatment volumes was achieved, with $\geq 95 \%$ of the CTV and PTV-F receiving 95\% of the prescribed dose in all trial patients. The PTV-I (median $798.9 \mathrm{~cm}^{3}$, 315.1-1265.5) was compromised by a median (range) of $4.0 \%(0.02-17.9)$ to exclude small bowel. The median V95 for the PTV-I was 97.5\% (80.1-99.9). Plans were optimized such that OAR doses were limited to commonly-accepted constraints for 5-fraction abdominal SABR [23, 24] as listed in Additional file 3: Table S2.

\section{Toxicity}

Patients were recruited to dose cohorts according to an $a$ priori dose-escalation scheme (Fig. 1). Demographic information, disease characteristics, and treatment details are listed by patient in Table 2. Overall, treatment was safe and well-tolerated. Table 3 lists all reported toxicity events that were possibly, probably, or definitely treatmentrelated. Three grade 3 events (possibly, probably, or definitely treatment-related) were reported: 2 fatigue (probably and definitely related) and 1 bone pain (possibly related). A large proportion of grade $1 \& 2$ toxicities were gastrointestinal, including altered taste, nausea, and vomiting (reported in $75 \%$ of patients). Fatigue $\geq$ grade 1 was reported in $50 \%$ of patients. All toxicities were effectively managed with supportive measures. There were no grade 4 or 5 treatment-related events. Patient 9 suffered an unrelated grade 4 pneumonia and patient 6 succumbed to unrelated grade 5 dyspnea secondary to pulmonary embolus.

\section{Renal Function}

Overall renal function was largely preserved following renal SABR. Nuclear medicine scans revealed reductions in the proportion of function of the ipsilateral kidney as well as in measured GFR in most patients (Fig. 2a). No significant reduction in GFR was observed at 12 weeks post-SABR [median 72.7 (range: $56.0-109.9)$ vs. $62.8(52.3-74.04) \mathrm{mL} /$ $\left.\mathrm{min} / 1.73 \mathrm{~m}^{2}, p=0.125\right]$. Likewise, pre- and post-SABR (at last follow-up) creatinine clearances were not significantly different [median 98.1 (range: 40.1-193) vs. 88.1 (53.1-139) $\mathrm{mL} / \mathrm{min}, p=0.164$ ] (Fig. 2b). No patients required dialysis.

\section{Quality of Life (QoL)}

Patient-reported quality of life was assessed by Functional Assessment of Cancer Therapy (FACT) questionnaires at baseline $(n=12)$ and at scheduled follow-up visits $(n=$ 10). QoL declined with respect to physical well-being ( $p=$ 0.016 ), but remained unchanged in the functional, social and emotional domains as well as overall QoL $(p>0.05)$. Likewise, QoL scores for a kidney-specific index (FACTKSI) were similar pre- and post-SABR $(p>0.05)$. Results are summarized in Additional file 3: Table S3.

Table 2 Summary of patient and disease characteristics by individual patient $(\mathrm{N}=12)$

\begin{tabular}{|c|c|c|c|c|c|c|c|c|c|c|c|c|c|}
\hline \multirow[t]{2}{*}{ Characteristic } & \multirow[t]{2}{*}{ Median $^{d}$} & \multicolumn{12}{|c|}{ Patient } \\
\hline & & 1 & 2 & 3 & 4 & 5 & 6 & 7 & 8 & 9 & 10 & 11 & 12 \\
\hline Age & 66.8 & 69 & 59 & 85 & 54 & 57 & 61 & 81 & 70 & 76 & 82 & 61 & 64 \\
\hline T Stage & - & $\mathrm{T} 3 \mathrm{c}$ & $\mathrm{T} 2 \mathrm{a}$ & $\mathrm{T} 3 \mathrm{~b}$ & $\mathrm{~T} 2 \mathrm{~b}$ & T3C & $\mathrm{T} 2 \mathrm{~b}$ & $\mathrm{~T} 2 \mathrm{a}$ & $\mathrm{T} 4$ & $\mathrm{~T} 2 \mathrm{a}$ & $\mathrm{T} 2 \mathrm{a}$ & Т3a & $\mathrm{T} 1 \mathrm{~b}$ \\
\hline N Stage & - & No & $N X$ & No & $N X$ & N1 & $N X$ & N1 & N1 & N1 & No & N1 & NO \\
\hline Laterality & - & $\mathrm{L}$ & $\mathrm{R}$ & $\mathrm{R}$ & $\mathrm{R}$ & $\mathrm{R}$ & $\mathrm{R}$ & $\mathrm{R}$ & $\mathrm{R}$ & $\mathrm{R}$ & $\mathrm{L}$ & $L$ & $L$ \\
\hline Tumor Size $(\mathrm{cm})^{a}$ & 8.7 & 9.3 & 7 & 8.9 & 11.9 & 11.2 & 13.8 & 7.3 & 10 & 7.9 & 7.5 & 9.8 & 4.8 \\
\hline Radiation Dose (Gy) / Fractions & - & $25 / 5$ & $25 / 5$ & $25 / 5$ & $30 / 5$ & $30 / 5$ & $30 / 5$ & $30 / 5$ & $30 / 5$ & $35 / 5$ & $30 / 5$ & $35 / 5$ & $35 / 5$ \\
\hline$\Delta \mathrm{CrCl}(\mathrm{mL} / \mathrm{min})$. & -13.5 & -13.5 & 35.9 & -12.1 & -4.6 & NR & -48.2 & $N R$ & NR & -36.6 & -24.7 & -54 & 17.5 \\
\hline Local Response $(\%)^{\mathrm{b}}$ & -17.3 & -11.8 & -17.1 & -30.3 & -25.2 & -25.9 & -5.8 & $N R$ & NR & $-54.4^{c}$ & +5.3 & -17.5 & -14.6 \\
\hline Time to Systemic Progression (months) & $3.9^{d}$ & 4.34 & 0.7 & 10.9 & 20.5 & 0.6 & 0.9 & 0.8 & 0.5 & 12.2 & 15.0 & 5.2 & 3.9 \\
\hline Systemic Therapy & - & $P$ & $P$ & - & $P, E$ & - & $\mathrm{T}$ & - & - & - & - & S & $S$ \\
\hline Survival (months) & $6.7^{d}$ & 4.9 & 10.2 & 16.4 & 43.6 & 1.5 & 3.2 & 1.3 & 2.4 & 13.6 & $22^{e}$ & 6.7 & 4.6 \\
\hline
\end{tabular}

L: left kidney, R: right kidney, HT - helical tomotherapy, IMRT: intensity modulated radiotherapy, VMAT: volumetric arc therapy, NR: not reported, CrCl: Creatinine clearance, $\boldsymbol{\Delta}$ - change, $P$ : pazopanib, $E$ : everolimus, $T$ : temsirolimus, $S$ : sunitinib. ${ }^{\text {a Longest tumor dimension on } C T \text { scan; }{ }^{b} M a x i m a l ~ r e d u c t i o n ~(-) ~ o r ~ i n c r e a s e ~(+) ~ i n ~}$ primary renal tumor size following SABR, expressed as percent of initial size; ${ }^{C} \mathrm{Pt} .9$ also underwent renal embolization (post-SABR) for refractory hematuria; ${ }^{\mathrm{d}}$ Median overall survival and time to systemic progression calculated using Kaplan-Meier method (95\% Cls: $1.48-16.43$ and $0.7-10.7$, respectively); ${ }^{e}$ Alive at last follow-up 
Table 3 All Possible Treatment-Related Toxicity Events ${ }^{\mathrm{a}}(\mathrm{N}=12)$

\begin{tabular}{|c|c|c|c|}
\hline Toxicity & Grade 1 & Grade 2 & Grade 3 \\
\hline Nausea & 13 & 3 & \\
\hline Vomiting & 4 & 1 & \\
\hline Altered Taste & 4 & & \\
\hline Anorexia & & 1 & \\
\hline Gas & & 2 & \\
\hline Diarrhea & & 2 & \\
\hline Constipation & 1 & & \\
\hline Dermatitis & 2 & 2 & \\
\hline Fatigue & 2 & 10 & 2 \\
\hline Pain - Abdominal & 5 & & \\
\hline Pain - Bone & 1 & & 1 \\
\hline Weakness & 2 & 1 & \\
\hline Lower Limb Edema & 4 & & \\
\hline Urinary $^{b}$ & 2 & & \\
\hline Insomnia & 2 & & \\
\hline Neuropathy & 1 & & \\
\hline Hot flashes & 3 & & \\
\hline Dizziness & & 1 & \\
\hline Headache & 3 & & \\
\hline
\end{tabular}

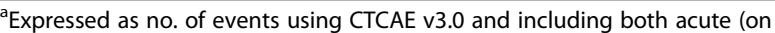
treatment) and late (reported in follow-up $\geq 4$ weeks post-SABR) ${ }^{\mathrm{b}}$ Frequency \& urgency

\section{Tumour Response and Overall Survival}

Despite local disease stability with a median tumour size reduction of $-17.3 \%$ (range: +5.3 to -54.4 ) at a median follow-up time of 5.3 months (95\% CI: 1.1-9.4), all patients progressed systemically (Fig. 3c). Overall survival (95\% CI) at 6,12 , and 24 months was $57.1 \%$ (25.4-79.6), 38.1\% (12.164.4), and 19.1\% (3.0-45.6), respectively (Fig. 3d). Median (95\% CI) overall survival was 6.7 months (1.5-16.4). Crude median follow-up (range) was 5.8 months (1.4-43.6).

\section{Discussion}

Here we report on the first prospective trial of renalablative SABR as an alternative to cytoreductive nephrectomy in non-operable patients with mRCC. Building on our institutional experience of this technique in a caseseries of unconventionally large renal tumours [22], this phase I trial evaluated cytoreductive SABR targeting the whole ipsilateral kidney, in essence to recapitulate the effects of debulking nephrectomy.

Our key findings include the safe delivery of 35 Gy in 5 fractions with minimal toxicity, favorable renal outcomes, preserved quality of life, and local disease stability in all cases where longitudinal imaging was available. Our trial design deliberately employed a conservative fractionation schedule so as to limit dose to organs at risk and thus minimize toxicity in this metastatic patient population

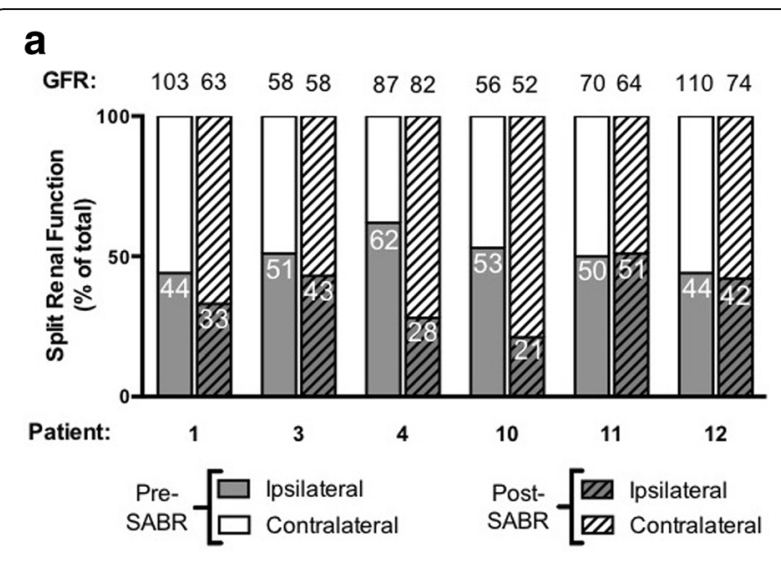

b

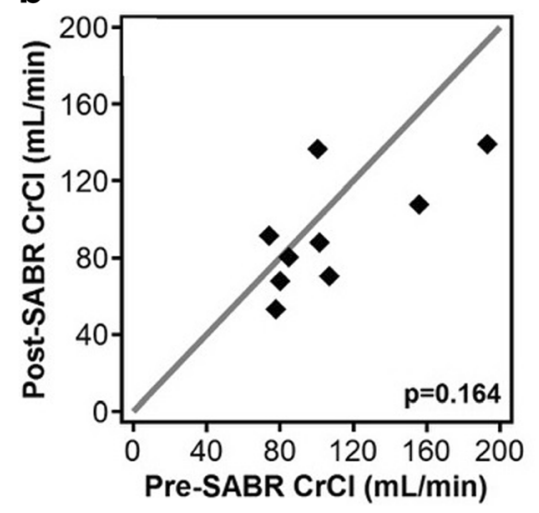

Fig. 2 Renal Function. a Functional contribution of both kidneys preand 12 weeks post-SABR with corresponding measured GFR values listed above each bar. Numbered bars indicate \% contribution from ipsilateral kidney. $\mathbf{b}$ Comparison of creatinine clearance $(\mathrm{CrCl})$ calculated using the Cockroft-Gault equation before and after stereotactic ablative radiotherapy (SABR) for patients with available pre- and post-SABR data $(n=9)$. $P$-value reported from the Wilcoxon signed-rank test

with poor PS. A five-fraction regimen is in keeping with multi-fraction SABR protocols which often use 3-5 fractions $[16,23]$. With respect to renal function, while no statistically significant decline was observed post-SABR, decreases in mean $\mathrm{CrCl}$ and GFR were noted $(-10 \mathrm{~mL} /$ $\mathrm{min}$ and $-9.9 \mathrm{~mL} / \mathrm{min} / 1.73 \mathrm{~m}^{2}$, respectively). These differences are relatively mild in the context of estimated decline in eGFR following radical nephrectomy: $22.4 \mathrm{~mL} / \mathrm{min} / 1.73 \mathrm{~m}^{2}$ based on a recent systematic review and meta-analysis (27 articles; 3719 patients) [26].

We hypothesized that primary renal SABR as an alternative to cytoreductive nephrectomy holds promise for several reasons. First, as tyrosine-kinase inhibitors (TKIs) are considered the standard of care in $\mathrm{mRCC}$, the initiation of these agents would be delayed following a nephrectomy owing to surgical healing. In contrast, SABR is a convenient non-invasive outpatient procedure that, in other disease sites, requires only a short delay (i.e. 2 weeks) prior to the initiation of a pharmacologic agent. In fact, one prospective study suggests that SABR to an extra-renal target with 


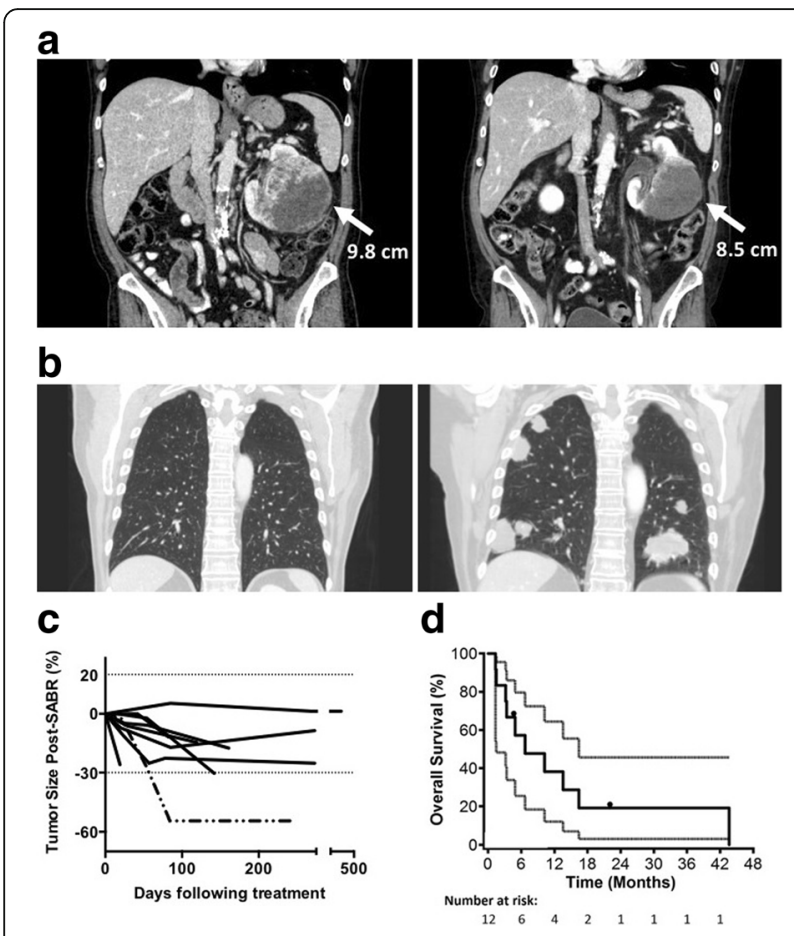

Fig. 3 Treatment Response and Survival. a Abdominal and $\mathbf{b}$ thoracic coronal $C T$ slices from patient 11 pre-SABR (left) and 7 months post-SABR (right). c Tumour size expressed as percent change in longest tumour dimension. Horizontal dotted lines mark a 20\% increase and 30\% decrease in size. Patient 9 (dotted/dashed line) also underwent renal embolization for refractory hematuria on day 27 post-SABR. d Kaplan-Meier plot of overall survival with $95 \%$ confidence bands $(n=12)$

concomitant pazopanib is safe [27]. Second, many primary targets in mRCC are technically inoperable due to tumour size or proximity to the collecting vessels and ureter. SABR can be targeted precisely to access any location within the affected kidney and dose can be sculpted within organ-at-risk tolerance. Third, SABR is inherently tissuesparing, whereas a nephrectomy may result in decline of pre-existing renal dysfunction or even de novo chronic kidney disease.

Finally, SABR holds promise for mRCC particularly in the era of contemporary immunotherapy. Despite the excitement surrounding checkpoint inhibition, response rates appear to reach a ceiling of 20-40\% [28]; for nivolumab, which has been approved for second-line treatment of $\mathrm{mRCC}$, the response rate is $25 \%$ [29]. Thus, it is essential to explore strategies to extend the benefits of these agents to additional patients. SABR has emerged as one such strategy since its mechanism of action partly involves inciting anti-tumour immune activation [30, 31] as well as abscopal (out-of-field) effects in mRCC and other malignancies [32, 33] - a phenomenon also thought to involve systemic anti-tumour immunity [19-21]. Interestingly, PD-1 inhibition can increase the frequency of radiotherapy-induced abscopal effects in a xenograft mouse model of RCC [20]. Clinically, several case reports have emerged demonstrating improved systemic responses when radiotherapy and checkpoint inhibition are combined [34-37]. Singh et al. recently demonstrated local anti-tumour immune activation in renal tumours following single-fraction SABR [38]. Our demonstration of the safety and tolerability of SABR in mRCC therefore justifies the combination SABR with immune checkpoint inhibition as a rational therapeutic strategy worthy of further investigation.

The limitations of this study include its small sample size at a single institution. Also, given the short median OS and consequently short follow-up in this trial (crude median follow-up: 5.8 months), the full range and severity of late toxicities may be under-appreciated. Another limitation is the heterogeneity in systemic treatment among the patients on this trial: specifically, the agents used and their timing was variable. Moreover, $50 \%$ of patients were not fit enough to receive systemic therapy. The OS of 6.7 months were relatively short and probably reflect, in part, the heterogeneity in patient selection and treatment; more careful patient selection and uniform treatment would likely be associated with better survival outcomes. Finally, it is unclear what the optimal local response assessment tool is in this setting, as distinguishing response from post-treatment effect remains challenging. The strengths of this study are its prospective design with a priori dose-escalation scheme and its novelty; to our knowledge, no other institution has evaluated post-SABR outcomes in the mRCC setting as we have done here.

\section{Conclusion}

We observed low toxicity with SABR to a dose of 35Gy in 5 fractions targeted to the primary tumour and ipsilateral kidney. Exploration of this regimen as an alternative to $\mathrm{CN}$ in larger prospective trials with up-front cytoreductive SABR followed by systemic therapy appears feasible based on our results. Additionally, tumour-targeted SABR could allow further dose-escalation while sparing normal renal parenchyma. Given the hypothesized immune activation induced by SABR, combining renal SABR with immune checkpoint inhibition in $\mathrm{mRCC}$ could be a particularly interesting approach and a multiinstitution phase II trial is planned.

\section{Additional files}

Additional file 1: Figure S1. Example of treatment planning volumes. To facilitate treatment of large PTVs, the initial PTV (teal outline) was trimmed in areas of overlap (green colorwash) with organs at risk - particularly small bowel (purple colorwash) - trimmed to generate a final PTV (red colorwash). (JPEG 127 kb)

Additional file 2: Figure S2. Clinical Course by Individual Patient. Time from initial radiologic diagnosis to death or last follow-up for each patient (y-axis intersect: SABR completion date). Duration and type of systemic 
therapy, additional local therapies, and date of systemic progression are indicated. 'Palliative embolization of bulky pelvic metastasis followed by hip replacement surgery; ${ }^{2}$ Palliative radiotherapy (20 Gy/5) following hip replacement; ${ }^{3}$ Renal artery embolization for refractory hematuria; ${ }^{4}$ Palliative radiotherapy to painful bony metastasis (shoulder). (JPEG $111 \mathrm{~kb}$ )

Additional file 3: Table S1. Target Dose and Volume Data by Individual Patient. CTV- clinical target volume; PTV-I - initial planning target volume; PTF-F - final planning target volume; NR - not reported. Table S2. Organ at Risk (OAR) Constraints and Doses (cGy). D1-99\% of the contoured volume receives this dose or less; D33-67\% of the contoured volume receives this dose or less: *Small bowel dose constraints were exceeded in Patients 2 \& 6 Patient 2 reported no toxicity whatsoever and Patient 6 reported only grade 1 emesis. Table S3. Summary of Quality of Life (QoL) Data at Baseline and Last Available Follow-Up $\left(N=12^{*}\right)$. (DOCX $\left.28 \mathrm{~kb}\right)$

\section{Abbreviations}

CN: Cytoreductive nephrectomy; CrCl: Creatinine clearance; CTCAE: Common Terminology Criteria for Adverse Events; eGFR: Estimated glomerular filtration rate; GFR: Glomerular filtration rate; Gy: Gray; IMDC: International Metastatic Renal Cell Carcinoma Database Consortium; IROCK: International Radiosurgery Oncology Consortium for Kidney; PRV: Planning risk volume; PS: Performance status; RCC: Renal cell carcinoma; SABR: Stereotactic ablative radiotherapy

\section{Acknowledgements}

We acknowledge medical physicists Dr. Jeff Chen and Dr. Slav Yartsev for assistance in retrieving dosimetric and treatment planning data for several trial patients.

\section{Funding}

Dr. Louie's research is supported by the Schulich Clinician Scientist Award.

\section{Availability of data and materials}

The datasets used in this study are available from the corresponding author on reasonable request.

\section{Authors' contribution}

Literature Search: RC, AL, BA, GR. Preparation of Ethics Documentation: BA, GR Study Design: BA, GR. Data Collection: BA, AL, MM, SP, GB, GR. Data Processing CJ, AW. Data Analysis: AW. Tables and Figures: AW and RC. Data Interpretation: AW, AL, RC. Manuscript Writing: RC, AL, AW. Review and Approval of Final Manuscript: All Authors.

\section{Ethics approval and consent to participate}

Ethics approval for this study was provided by the Western University Human Studies Research Ethics Board (REB\# 15680). All study participants provided written consent to participate in this trial.

\section{Consent for publication}

All study participants provided written consent which included consent for the publication of trial results.

\section{Competing interests}

Dr. Louie has received speaker's honoraria from Varian Medical Systems, Inc

\section{Publisher's Note}

Springer Nature remains neutral with regard to jurisdictional claims in published maps and institutional affiliations.

\section{Author details}

${ }^{1}$ Department of Radiation Oncology, London Regional Cancer Program, London, Canada. ${ }^{2}$ Department of Medical Oncology, London Regional Cancer Program, London, Canada. ${ }^{3}$ Division of Urology, Western University, London, Canada. ${ }^{4}$ Division of Surgical Oncology, Western University, London, Canada. ${ }^{5}$ Department of Epidemiology and Biostatistics, Western University, London, Canada.
Received: 6 January 2018 Accepted: 6 March 2018

Published online: 20 March 2018

\section{REFERENCES}

1. Flanigan RC, Mickisch G, Sylvester R, Tangen C, Van Poppel H, Crawford ED. Cytoreductive nephrectomy in patients with metastatic renal cancer: a combined analysis. J Urol. 2004:171:1071-6.

2. Hanna N, Sun M, Meyer CP, et al. Survival Analyses of Patients With Metastatic Renal Cancer Treated With Targeted Therapy With or Without Cytoreductive Nephrectomy: A National Cancer Data Base Study. Journal of clinical oncology : official journal of the American Society of Clinical Oncology. 2016:34:3267-75.

3. Petrelli F, Coinu A, Vavassori I, et al. Cytoreductive Nephrectomy in Metastatic Renal Cell Carcinoma Treated With Targeted Therapies: A Systematic Review With a Meta-Analysis. Clin Genitourin Cancer. 2016; 14:465-72.

4. Choueiri TK, Xie W, Kollmannsberger C, et al. The impact of cytoreductive nephrectomy on survival of patients with metastatic renal cell carcinoma receiving vascular endothelial growth factor targeted therapy. J Urol. 2011:185:60-6.

5. Bex A. Immediate versus deferred cytoreductive nephrectomy (CN) in patients with synchronous metastatic renal cell carcinoma (mRCC) receiving sunitinib (EORTC 30073 SURTIME). Madrid: ESMO 2017 Congress; 2017.

6. Tsao CK, Small AC, Moshier EL, et al. Trends in the use of cytoreductive nephrectomy in the United States. Clin Genitourin Cancer. 2012;10:159-63.

7. Conti SL, Thomas IC, Hagedorn JC, et al. Utilization of cytoreductive nephrectomy and patient survival in the targeted therapy era. International journal of cancer Journal international du cancer. 2014;134:2245-52.

8. Abdollah F, Sun M, Thuret R, et al. Mortality and morbidity after cytoreductive nephrectomy for metastatic renal cell carcinoma: a population-based study. Ann Surg Oncol. 2011;18:2988-96.

9. Ning S, Trisler K, Wessels BW, Knox SJ. Radiobiologic studies of radioimmunotherapy and external beam radiotherapy in vitro and in vivo in human renal cell carcinoma xenografts. Cancer. 1997;80:2519-28.

10. Ponsky LE, Crownover RL, Rosen MJ, et al. Initial evaluation of Cyberknife technology for extracorporeal renal tissue ablation. Urology 2003:61:498-501.

11. Walsh L, Stanfield JL, Cho LC, et al. Efficacy of ablative high-dose-perfraction radiation for implanted human renal cell cancer in a nude mouse model. Eur Urol. 2006;50:795-800. discussion.

12. Kothari G, Foroudi F, Gill S, Corcoran NM, Siva S. Outcomes of stereotactic radiotherapy for cranial and extracranial metastatic renal cell carcinoma: a systematic review. Acta Oncol. 2015:54:148-57.

13. Alongi $F$, Arcangeli $S$, Triggiani $L$, et al. Stereotactic ablative radiation therapy in renal cell carcinoma: From oligometastatic to localized disease. Crit Rev Oncol Hematol. 2017:117:48-56.

14. Siva S, Pham D, Gill S, Corcoran NM, Foroudi F. A systematic review of stereotactic radiotherapy ablation for primary renal cell carcinoma. BJU Int. 2012;110:E737-43

15. Siva S, Kothari G, Muacevic A, et al. Radiotherapy for renal cell carcinoma: renaissance of an overlooked approach. Nature reviews. Urology. 2017;14: 549-563.

16. Siva S, Muacevic A, Staehler M, et al. Individual Patient Data Meta-analysis of SBRT Kidney: A Report From the International Radiosurgery Oncology Consortium for Kidney (IROCK). International Journal of Radiation Oncology*Biology*Physics. 2017:99:S153-S4.

17. Shin BJ, Chick JF, Stavropoulos SW. Contemporary Status of Percutaneous Ablation for the Small Renal Mass. Curr Urol Rep. 2016;17:23.

18. Kurup AN. Percutaneous ablation for small renal masses-complications. Semin Interv Radiol. 2014;31:42-9.

19. Demaria $\mathrm{S}, \mathrm{Ng} \mathrm{B}$, Devitt $\mathrm{ML}$, et al. Ionizing radiation inhibition of distant untreated tumors (abscopal effect) is immune mediated. Int J Radiat Oncol Biol Phys. 2004:58:862-70.

20. Park SS, Dong H, Liu X, et al. PD-1 Restrains Radiotherapy-Induced Abscopal Effect. Cancer immunology research. 2015;3:610-9.

21. Brooks ED, Schoenhals JE, Tang C, et al. Stereotactic Ablative Radiation Therapy Combined With Immunotherapy for Solid Tumors. Cancer J. 2016;22:257-66

22. Correa RJ, Rodrigues GB, Chen H, Warner A, Ahmad B, Louie AV. Stereotactic Ablative Radiotherapy (SABR) for Large Renal Tumors: A Retrospective Case 
Series Evaluating Clinical Outcomes, Toxicity, and Technical Considerations. Am J Clin Oncol. 2016. Epub ahead of print.

23. Siva S, Ellis RJ, Ponsky L, et al. Consensus statement from the International Radiosurgery Oncology Consortium for Kidney for primary renal cell carcinoma. Future Oncol. 2016;12:637-45.

24. Timmerman RD. An overview of hypofractionation and introduction to this issue of seminars in radiation oncology. Semin Radiat Oncol. 2008;18:215-22.

25. Heng DY, Xie W, Regan MM, et al. Prognostic factors for overall survival in patients with metastatic renal cell carcinoma treated with vascular endothelial growth factor-targeted agents: results from a large, multicenter study. Journal of clinical oncology : official journal of the American Society of Clinical Oncology. 2009;27:5794-9.

26. Patel HD, Pierorazio PM, Johnson MH, et al. Renal Functional Outcomes after Surgery, Ablation, and Active Surveillance of Localized Renal Tumors: A Systematic Review and Meta-Analysis. Clin J Am Soc Nephrol. 2017;12:10571069.

27. De Wolf $\mathrm{K}$, Rottey $\mathrm{S}$, Vermaelen $\mathrm{K}$, et al. Combined high dose radiation and tyrosine kinase inhibitors in renal cell carcinoma: a phase I trial. ESTRO Annual Meeting. Vienna, Austria. Radiother Oncol. 2017;12:157.

28. Demaria S, Coleman CN, Formenti SC. Radiotherapy: Changing the Game in Immunotherapy. Trends Cancer. 2016;2:286-94.

29. Motzer RJ, Escudier B, McDermott DF, et al. Nivolumab versus Everolimus in Advanced Renal-Cell Carcinoma. N Engl J Med. 2015;373:1803-13.

30. Lugade AA, Moran JP, Gerber SA, Rose RC, Frelinger JG, Lord EM. Local radiation therapy of B16 melanoma tumors increases the generation of tumor antigen-specific effector cells that traffic to the tumor. J Immunol. 2005;174:7516-23.

31. Kim MS, Kim W, Park IH, et al. Radiobiological mechanisms of stereotactic body radiation therapy and stereotactic radiation surgery. Radiation oncology journal. 2015;33:265-75.

32. Wersall PJ, Blomgren H, Pisa P, Lax I, Kalkner KM, Svedman C. Regression of non-irradiated metastases after extracranial stereotactic radiotherapy in metastatic renal cell carcinoma. Acta Oncol. 2006:45:493-7.

33. Siva S, MacManus MP, Martin RF, Martin OA. Abscopal effects of radiation therapy: a clinical review for the radiobiologist. Cancer Lett. 2015;356:82-90.

34. Golden EB, Demaria S, Schiff PB, Chachoua A, Formenti SC. An abscopal response to radiation and ipilimumab in a patient with metastatic nonsmall cell lung cancer. Cancer immunology research. 2013;1:365-72.

35. Hiniker SM, Chen DS, Reddy S, et al. A systemic complete response of metastatic melanoma to local radiation and immunotherapy. Transl Oncol. 2012;5:404-7.

36. Postow MA, Callahan MK, Barker CA, et al. Immunologic correlates of the abscopal effect in a patient with melanoma. N Engl J Med. 2012;366:925-31.

37. Reynders K, Illidge T, Siva S, Chang JY, De Ruysscher D. The abscopal effect of local radiotherapy: using immunotherapy to make a rare event clinically relevant. Cancer Treat Rev. 2015:41:503-10.

38. Singh AK, Winslow TB, Kermany MH, et al. A Pilot Study of Stereotactic Body Radiation Therapy Combined with Cytoreductive Nephrectomy for Metastatic Renal Cell Carcinoma. Clin Cancer Res. 2017;23:5055-5065.

\section{Submit your next manuscript to BioMed Central and we will help you at every step:}

- We accept pre-submission inquiries

- Our selector tool helps you to find the most relevant journal

- We provide round the clock customer support

- Convenient online submission

- Thorough peer review

- Inclusion in PubMed and all major indexing services

- Maximum visibility for your research

Submit your manuscript at www.biomedcentral.com/submit

CBiomed Central 